\section{It's porin' CNTs}

Lipid-coated carbon nanotubes can insert themselves spontaneously into lipid bilayers and live-cell membranes.

Carbon nanotubes (CNTs), which are cylindrical layers of graphene in which carbon bonds are arranged in a hexagonal pattern, are a highly versatile and valuable nanomaterial. The characteristics of CNTs, such as their thermal and electrical conductance, make them a material of choice for a variety of uses, including in computers and bioimaging. Adding an important biological application to the list, Aleksandr Noy at the Lawrence Livermore National Laboratory and his colleagues recently reported that CNTs can also function as pores in a lipid bilayer, permitting the transport of water, ions and DNA.

The first challenge Noy and his colleagues faced was stabilizing the CNTs in solution, which they did using lipids. They then sonicated the CNTs to cut them into small fragments and obtained short tubes of about 5-15 nanometers in length. These short CNTs incorporated spontaneously into liposomes, perpendicularly to the membrane plane. Whereas some CNTs matched the length of the lipid bilayer, longer ones interacted with hydrophilic head groups at one end of the bilayer and stuck out into the solution at the other end. "We thought the CNT length would have to match exactly the lipid bilayer, but CNTs of other sizes work, too," Noy says.

And work well they do-the scientists showed that the CNT porins, as they call them, can transport ions, causing shrinkage of water-filled liposomes when placed in a high-salt solution. The CNT porins also transport single-stranded DNA at an average speed of 1.5 nucleotides per millisecond, which is compatible with nanopore sequencing applications. The team further demonstrated that these synthetic pores spontane- ously incorporate into the membranes of live mammalian cells. Using patch-clamp measurements, they found that the CNT porins mimic in cells the conductance dynamics they show in planar lipid bilayers.

Although Noy admits that applications of CNT porins may be still far off in the future, he points out that "the CNT ends could be functionalized and used to make cells permeable to a particular element." CNT porins could be useful in the treatment of human diseases involving ion channel defects and for drug delivery to specific tissues. In the laboratory, CNT porins can potentially be used in a range of applications, such as emerging sequencing technologies, nanosensors and synthetic cellular interfaces.

\section{Irene Jarchum}

\section{RESEARCH PAPERS}

Geng, J. et al. Stochastic transport through carbon nanotubes in lipid bilayers and live cell membranes. Nature 514, 612-615 (2014). 